\title{
SARCOPENIA OF AGEING: FUNCTIONAL, STRUCTURAL AND BIOCHEMICAL ALTERATIONS
}

\author{
Zhong S, Chen CN \& Thompson LV \\ University of Minnesota, Minneapolis, Minnesota - USA \\ Correspondence to: LaDora V. Thompson, PhD, PT, University of Minnesota, Program in Physical Therapy, 420 \\ Delaware Street, S.E., Minneapolis, MN 55455 - USA, e-mail: thomp067@umn.edu
}

Recebido: 19/02/2007 - Revisado: 19/03/2007 - Aceito: 21/03/2007

\begin{abstract}
Aging is associated with a progressive decline of muscle mass, strength, and quality, a condition described as sarcopenia of aging. Despite the significance of skeletal muscle atrophy, the mechanisms responsible for the deterioration of muscle performance are only partially understood. The purpose of this review is to highlight cellular, molecular and biochemical changes that contribute to age-related muscle weakness.

Key words: age, skeletal muscle fiber, myosin ATPase, myofibril.
\end{abstract}

\section{RESUMO}

\section{Sarcopenia no envelhecimento: Alterações funcionais, estruturais e bioquímicas}

O envelhecimento está associado ao declínio progressivo da massa, força, e qualidade muscular, uma condição descrita como sarcopenia do envelhecimento. Apesar da importante atrofia do músculo esquelético, os mecanismos responsáveis pela deterioração do desempenho muscular são somente parcialmente conhecidos. A proposta desta revisão é ressaltar as alterações celulares, moleculares e bioquímicas que contribuem para a fraqueza muscular associada ao envelhecimento.

Palavras-chave: envelhecimento, fibra músculo esquelética, miosina ATPase, miofibrila. 


\section{SARCOPENIA}

Aging is associated with a progressive decline of muscle mass, strength, and quality, a condition described as sarcopenia of aging. Interestingly, these age-related changes are observed among healthy, physically active adults with the rate of muscle loss at $1-2 \%$ per year past the age of $50^{1}$. The prevalence of sarcopenia is about $25 \%$ of individuals under the age of 70 years and $40 \%$ of those aged 80 years or older ${ }^{2}$.

Sarcopenia represents a risk factor for frailty, loss of independence, and physical disability ${ }^{3}$. Impaired muscle strength is predictive of disability and all-cause mortality in advanced age ${ }^{4,5}$. Loss of mobility resulting from muscle loss predicts major physical disability and mortality, and is associated with poor quality of life, social needs, and health care needs ${ }^{6}$. The economic impact of sarcopenia and its detrimental correlates is immense ${ }^{7}$. Thus, understanding the mechanisms leading to muscle weakness and physical disability at advanced age represents a high public health priority. Epidemiological studies suggest multiple contributing factors, including neuronal and hormonal changes, inadequate nutrition, low-grade chronic inflammation, and physical inactivity ${ }^{8-11}$. The purpose of this review is to highlight cellular, molecular, and biochemical changes that contribute to age-related muscle weakness.

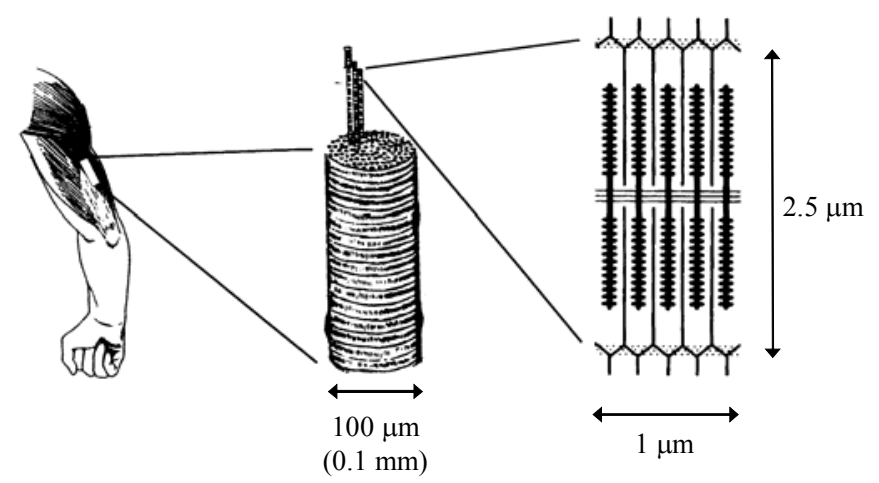

Muscle

Fiber

Sarcomere

Figure 1. Skeletal muscle structure. The skeletal muscle is composed of individual muscle fibers arranged in parallel. An individual muscle fiber is composed of myofibrils arranged in parallel. The myofibril is made up of the major contractile unit, the sarcomere. Sarcomeres are arranged in series to form a single myofibril. With aging, the individual fibers decrease in cross-sectional area by decreasing the number of myofibrils.

\section{Muscle physiology}

Skeletal muscles are composed of hundreds to hundreds of thousands of individual, elongated, multinucleated cells called fibers. An individual muscle fiber contains a bundle of myofibrils, each being a linear array of sarcomeres. The number of fibers in a given muscle is determined at birth and changes little throughout the life span except in cases of injury or disease. In contrast, the number of myofibrils, and, consequently muscle fiber cross-sectional area, can change dramatically, increasing with normal growth or hypertrophy induced by strength training and decreasing with atrophy associated with immobilization, disease, or old age (Figure 1).

The sarcomere, the primary contractile unit, is composed of many proteins. Myosin (thick filaments), actin (thin filaments), and regulatory proteins (troponin and tropomyosin) are critical for muscle function. The interactions of the myosin head with actin in the presence of ATP during the actomyosin ATPase cycle results in sliding of thin filaments past thick filaments toward the center of the sarcomere. The biochemical steps of ATP hydrolysis during this cyclic interaction of actin with myosin are accompanied by a sequence of structural transitions in both proteins. The physiologically relevant step of force generation is a transition of the actomyosin complex from the states of weak interaction (A.M.ATP and A.M.ADP.P ) to the states of strong interaction (AM.ADP and $\mathrm{AM})^{8-12}$. In other words, force generation occurs when myosin and actin are in the strongbinding structural state (Figure 2). Thus, age-related structural or chemical changes in actin and myosin that affect the weakto-strong actomyosin transition are potential candidates contributing to sarcopenia.

\section{Age-related changes in muscle}

Despite the significance of skeletal muscle atrophy and weakness as inevitable concomitants of old age, the

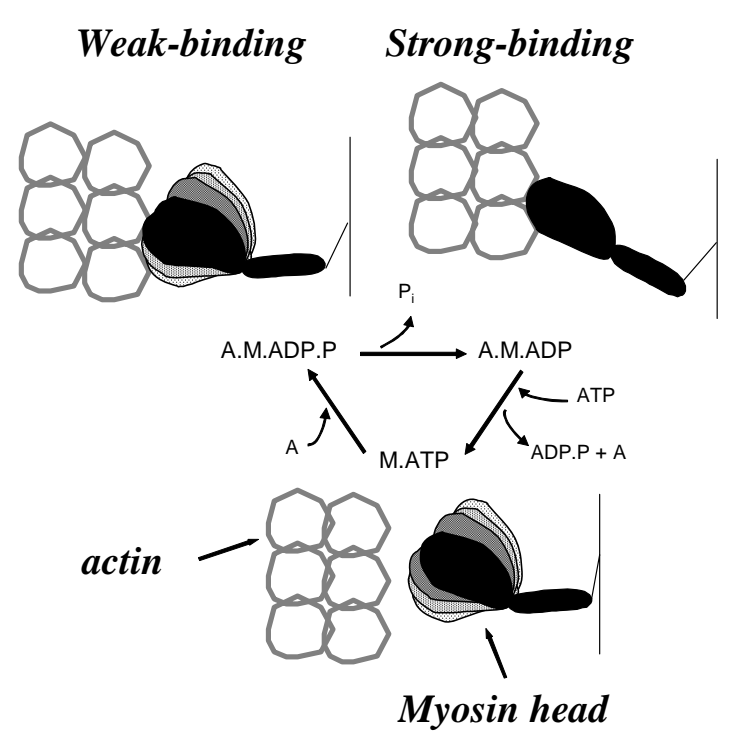

Figure 2. Structural changes of the myosin head during muscle contraction, coupled to the ATPase cycle. Force is produced when the myosin head changes from a weak-binding structure, in which the catalytic domain is dynamically disordered, to a strong-binding structure, in which the head is rigid. The structure of the myosin head is altered in an agedependent manner. 
mechanisms responsible for these impairments are only partially understood. Age-associated muscle atrophy is the result of a combination of individual-fiber atrophy, along with a decrease in the total number of fibers, with a preferential loss of type II (fast-twitch, glycolytic, fibers with myosin heavy chain type II isoforms) muscle fibers ${ }^{13,14}$. Fiber number appears to decrease as a result of the loss of whole motor units. For gastrocnemius muscles of rats, the number of fastfatigable (FF) units decrease in old age by approximately onethird compared with adult values ${ }^{15}$. In contrast, the number of slow (S) units remain constant with aging, but the number of fibers per motor unit increase threefold in old age ${ }^{15}$. The maximum force of FF motor units in old rats is $70 \%$ that of comparable motor units in adult rats, whereas that of $\mathrm{S}$ motor units is $250 \%$ of the adult value. On the basis of these data, the conclusion is that the age-related remodeling of motor units involves selective denervation of fast muscle fibers with reinnervation of some of the denervated fibers by axonal sprouting from slow fibers. The net result of the loss and atrophy of individual fibers and the loss of fast motor units with increasing size of slow motor units includes muscle weakness and loss of fine movement.

Finally, in addition to the loss in muscle mass and consequent loss in muscle force, muscles of old animals show a deficit of $20 \%$ in specific force (force generation normalized for muscle cross-sectional area), suggesting qualitative as well as quantitative deficiencies associated with aging ${ }^{14,16}$. Moreover, aging is associated with a progressive slowing of muscle movement ${ }^{13}$. The mechanisms of agerelated deterioration of contractility involve multiple factors associated with changes in the process of muscle excitationcontraction coupling.

\section{Age-related changes in contractility}

Age-related deterioration of muscle contractility is observed at the whole muscle level and at the single fiber level. Single skeletal muscle fiber contractility is determined in two experimental preparations, the intact and the permeabilized muscle fiber preparations. The intact single muscle fiber has a functional membrane-bound excitation-contraction coupling system whereas the permeabilized fiber lacks this system and the proteins involved in energy metabolism. The intact fiber offers information of two protein complexes critical for effective excitation-contraction coupling: dihydropyridine and ryanodine receptors. In contrast, the permeabilized fiber offers more direct information on the contractile proteins (myosin and actin). Literature supports excitation-contraction coupling contributing to age-related decline in muscle contractility. In particular, there are decreases in dihydropyridine and ryanodine receptors that translate membrane depolarization into intracellular $\mathrm{Ca}^{2+}$ release, a decrease in the dihydropyridine and ryanodine receptors ratio, and a decrease in functional dihydropyridine receptors leading to excitation-contraction uncoupling in old skeletal muscle ${ }^{17}$. Investigations focused on the permeabilized individual muscle fiber show that the two principal contractile parameters, specific force $\left(\mathrm{P}_{\mathrm{o}}\right)$ and unloaded shortening velocity $\left(\mathrm{V}_{\mathrm{o}}\right)$, decrease progressively with age. A close review of the literature reveals that the extent of deterioration depends on many factors, such as the fiber type composition of the specific muscle studied, the selected age group of the rats and the rodent strain (life-span). For instance, in single permeabilized myosin heavy chain type I isoform fibers from the soleus muscle, aging to 20-37 months produce a $10 \%$ to $47 \%$ decline in $\mathrm{P}_{\mathrm{o}}$ and a $33 \%$ to $49 \%$ decline in $\mathrm{V}_{\mathrm{o}}{ }^{14,18,19}$. In contrast, in single permeabilized myosin heavy chain type IIB isoform fibers from the semimembranosus muscle, aging to 28-36 months produce a $20-30 \%$ decline in both $\mathrm{P}_{\mathrm{o}}$ and $\mathrm{V}_{\mathrm{o}}{ }^{20-22}$. The experimental results from rodent studies parallel the findings from human biopsy studies. Single permeabilized fibers from human vastus lateralis muscle reveal an age-related decrease in contractility, with $4 \%$ to $28 \%$ decrease in $\mathrm{P}$ and $7 \%-28 \%$ decrease in $\mathrm{V}_{0}{ }^{23-26}$. On the basis of the single fiber data, the decline of contractility supports age-related changes in myosin and/or actin.

\section{Age-related structural changes in myosin and actin}

Electron paramagnetic resonance (EPR) is a highresolution spectroscopic method which, in combination with site-specific spin labeling of $\mathrm{Cys}_{707}$ of myosin, detects changes in the structure of myosin associated with relaxation and contraction of muscle fibers. Specifically, EPR can be used to determine quantitatively the fraction of myosin molecules in the weak- and strong-binding structural states. EPR studies show that the age-related $24 \%-27 \%$ decrease in the specific force is associated with $24 \%-30 \%$ decrease in the fraction of myosin heads in the strong-binding, force-generating structural state ${ }^{12,20,22,27}$. In other words, there are structural changes in myosin during contraction with age (Figure 2).

\section{Age-related changes in actomyosin ATPase activity and in functional properties of myosin and actin}

Hydrolysis of ATP is directly related to contractile function and structural changes in the head region of myosin during contraction (Figure 2). In particular, myosin ATPase activity is necessary for the generation and maintenance of force. From an experimental perspective, myofibrils which preserve the organization and interaction between the contractile proteins are often used to determine ATPase activity. Yet purified actin and myosin can be used to determine ATPase activity too, permitting the determination of enzymatic changes in each of these proteins, independently of the other and without interference from other myofibrillar proteins.

Myosin ATPase activity can be determined at high and low salt concentrations, revealing critical information about the proteins. Myofibrillar ATPase activity at high salt concentration eliminates effects of actin and other proteins, and is sensitive to post-translational changes in $\operatorname{myosin}^{23}$. Age- 
related molecular changes in myosin are observed in highsalt ATPase activities of myofibrils and myosin ${ }^{18-21}$. Like single muscle fibers described previously, the age-induced changes are muscle-specific and strain-dependent. This data suggests that post-translational modification of myosin occurs with age.

Although myofibrillar ATPase activity at high salt concentration provides critical information about myosin, it is not physiological. Physiological ATPase depends on the state of myosin as well as actin and is directly associated with $\mathrm{V}_{0}$. Physiological ATPase can be determined in myofibrils or in purified myosin and actin. Under isometric conditions, simultaneous measurements of force and ATPase activity in single fibers of aged (32 - 37 months) and young (8-12 months) rats show that fibers from aged rats generate $\sim 20 \%$ lower maximum force without changes in the ATPase activity $^{23,28}$. This result indicates a decrease in the energetic efficiency, a partial uncoupling between ATPase activity and force generation, during contraction in aged muscle. Under unloaded shortening conditions in the same muscle, aging to 32 - 38 months results in a 16\% decrease in the myofibrillar ATPase activity and a similar decrease in the shortening velocity ${ }^{29}$. This data suggests that changing actin-myosin interactions contribute to age-related inhibition of contractility.

Biochemical experiments on purified actin and myosin from young (4 - 12 months) and aged (27 - 35 months) rats show a decrease in two parameters of the actomyosin ATPase, $\mathrm{V}_{\max }$ (activity extrapolated to infinite actin concentration) and $\mathrm{K}_{\mathrm{m}}$ (the concentration of actin at half $\mathrm{V}_{\max }$ ) (Figure 3$)^{21,23}$. Subsequent mixing of actin and myosin from young and old

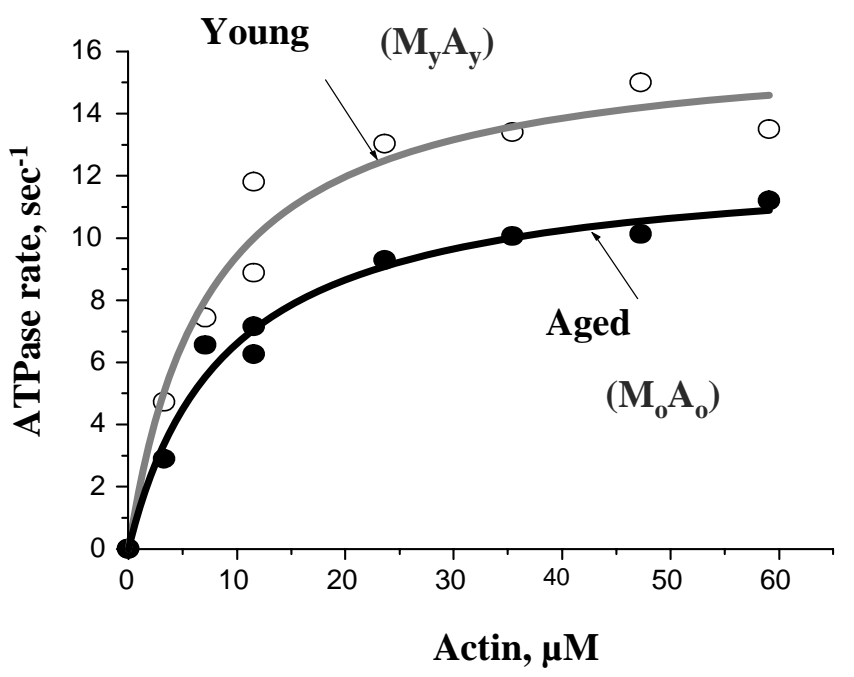

Figure 3. Representative experiment with isolated myosin and actin proteins from young and old rats to determine Vmax (the maximum rate) and $\mathrm{Km}$ (actin concentration at half Vmax). The actin-activated myosin ATPase rate was measured at increasing concentrations of actin. The $\mathrm{Vmax}$ and $\mathrm{Km}$ were determined with $\mathrm{O}=$ young myosin, young actin, $\mathbf{M}_{\mathrm{y}} \mathbf{A}_{\mathrm{y}}, \mathrm{Vmax}=16.43 \pm 1.00 \mathrm{~s}^{-1}, \mathrm{Km}=7.44 \pm 1.63 \mu \mathrm{M} ; \bullet \bullet=$ old myosin, old actin, $\mathbf{M}_{\mathbf{o}} \mathbf{A}_{\mathbf{o}}, \mathrm{Vmax}=12.55 \pm 0.62 \mathrm{~s}^{-1}, \mathrm{Km}=8.98 \pm 1.48 \mu \mathrm{M}$. muscle in four combinations shows that the age-related decrease in $\mathrm{V}_{\max }$ is primarily due to changes in myosin. This decrease in $\mathrm{V}_{\text {max }}$ is consistent with our earlier study showing age-related alterations in the structural states of myosin (transitions from weak to strong interactions, Figure 2). Yet, the age-related decrease in $\mathrm{K}_{\mathrm{m}}$ is due to changes in both proteins, as actin from young muscle attenuates the age-related decrease in $\mathrm{K}_{\mathrm{m}}$ for myosin from old muscle. The changes in $\mathrm{K}_{\mathrm{m}}$ are related to changes in the equilibria between actin and myosin-nucleotide complexes at the final stages of the cycle. The data from these experiments suggests that changes in actin, together with changes in myosin, are involved in the molecular mechanism of age-related deterioration of muscle contractility.

\section{Age-related oxidative modifications of skeletal muscle proteins}

The 'free radical theory' of aging, formulated 50 years ago, proposes that aging and associated degenerative diseases can be attributed to deleterious effects of reactive oxygen species $^{30}$. A current version of this theory is the 'oxidative stress theory' of aging. The 'oxidative stress theory' states that "A chronic state of oxidative stress exists in cells of aerobic organisms even under normal physiological conditions because of an imbalance of proxidants and antioxidants. This imbalance results in a steady-state accumulation of oxidative damage in a variety of macromolecules. Oxidative damage increases during aging, which results in a progressive loss in the functional efficiency of various cellular processes ${ }^{31}$ ".

Oxidative stress is thought to play a significant role in sarcopenia by modifying cysteine and tyrosine residues, leading to a loss of key skeletal muscle function. For instance, the muscle protein most intensively studied is the sarcoplasmic/ endoplasmic-reticulum $\mathrm{Ca}^{2+}$-ATPase, SERCA. These investigations focus on what sites are vulnerable to oxidative stress, and how the modification alters protein function with increasing age. Normal aging of skeletal muscle is associated with increased nitration; in particular, specific nitration of the SERCA2a isoform in slow-twitch muscle ${ }^{32,33}$. This modification increases by at least 3 -fold with age in skeletal muscle, and correlates with a $40 \%$ loss in Ca-ATPase activity. Subsequent mass spectrometry analysis reveals an agedependent accumulation of 3-nitrotyrosine at positions 294 and 295 of the SERCA2 protein, suggesting that these vicinal tyrosines play a critical role in muscle function In vitro studies also demonstrate that SERCA2a is inherently sensitive to tyrosine nitration with concomitant functional deficits ${ }^{32,33}$. Because the physiological role of the Ca-ATPase is to mediate muscle relaxation, the consequence of nitration-induced inhibition of SERCA2a most likely explains the slower contraction and relaxation times observed in skeletal muscle from old animals.

Aging also leads to a partial loss of SERCA1 isoform activity, and a molecular rationale for this phenomenon may 
be the age-dependent oxidation of specific cysteine residues. A quantitative mapping of the specific cysteine residues reveals nine cysteine residues targeted by age-dependent oxidation in vivo, and six cysteine residues partially lost upon oxidant treatment of the SR in vitro ${ }^{34}$, although the residues affected in vivo do not completely match those targeted in vitro, suggesting that modification of some residues appears not to contribute significantly to the age-dependent loss of SERCA function. Taken together, these studies provide some insights about the molecular mechanisms responsible for age-related alterations in calcium regulation in skeletal muscle.

In the presence of reactive oxygen and nitrogen species, force is inhibited and calcium sensitivity is altered in single skeletal muscle fibers ${ }^{35-37}$. In vivo oxidative modifications of myosin and actin focus on selective markers of oxidative damage, such as carbonylation, nitration, formation of HNE (4-hydroxy-2-nonenal) adducts, and oxidation of cysteines ${ }^{38}$. 3 -nitrotyrosine and HNE-adducts are detected in actin and in myosin, but in both cases the level of modification is similar in young and old muscle. Although this finding does not implicate accumulation of these two oxidative stress markers (oxidative stress theory of aging), it does not eliminate sitespecific modifications of key amino acids which are critical for function.

An age-related decrease in cysteine content is detected in myosin, but cysteine content of actin is unaffected by age ${ }^{21}$. Thus, accumulation of these modifications are unlikely to explain previously described age-related inhibitory changes in this muscle's contractility and actomyosin interactions ${ }^{20-}$ ${ }^{22}$. The implication of this finding for muscle contractility depends on the still unknown localization of post-translationally modified sites. Oxidation of one or two reactive myosin cysteines (Cys 707 and Cys 696) could result in significant deterioration of muscle contractility ${ }^{23}$, but myosin contains about 40 cysteines, and the functional role of the majority is not known.

Another possible explanation of age-related inhibitory effects on myosin is glycation, which has been detected in the skeletal muscle of aged rats ${ }^{39}$. In vitro studies on myosin purified from muscle of young rats reveal that glycation of myosin decreases actin motility ${ }^{40}$. It has been proposed that the mechanism of this functional loss is modification of lysinerich nucleotide- and actin-binding regions of myosin ${ }^{40}$. We recently demonstrated an increase in glycation in skeletal muscle tissues from diabetic rats, with the accumulation occurring within the myofibers ${ }^{41}$. Future identification of the modified proteins will likely assist in determining whether glycation plays a role in contractile dysfunction with disease and aging.

An important limitation in the characterization of modified proteins from aged tissue is the fact that the data provide only a snapshot of a dynamic process, as proteins are constantly being synthesized and degraded in most tissues. Furthermore, current knowledge about post-translational modification, and the techniques available to measure them, may not permit the quantitative analysis of all potential post-translational modifications of a given protein of interest as well as its functional characterization.

\section{PHYSICAL THERAPY IMPLICATIONS}

Physical therapists frequently work with the older adult or the elderly. The elderly usually come to the physical therapy clinic due to neurological problems (such as poor balance) or musculo-skeletal problems (such as fracture, joint replacement and arthritis) instead of purely muscle atrophy and weakness. Yet, muscle strength is critical for the older adult or elderly to regain or maintain independence in activities of daily living. Trained as physical therapists, we know muscle strength can be improved by resistance exercise while aerobic exercise has only negligible effects on muscle strength. In addition, there is research demonstrating the effect of resistance exercise on muscle strength is similar between the elderly and young adults ${ }^{42}$.

However, with more and more evidence that links aging with oxidative stress and sarcopenia, we need to know how exercise, the frequently used intervention, impacts oxidative stress and muscle strength. To date, the debate about whether resistance exercise is good or not is still going. Some studies show positive effect of resistance exercise on oxidative stress and muscle strength while others do not. With the knowledge in cellular, molecular and biochemical changes of aging muscles, physical therapists can keep updating the related information from the still ongoing studies. More importantly, physical therapists therefore can apply the latest knowledge from research to practice.

\section{CONCLUSION}

Clearly, physiological studies detect age-related deterioration of contractility of single fibers. Structural analysis, using EPR, shows structural changes in myosin which are correlated with the age-related decline in force. Biochemical studies reveal age-related molecular changes in myosin and actin, which are most likely due to oxidative modifications. In view of the muscle-specificity of age-related changes, understanding of the molecular basis of physiological changes contributing to sarcopenia requires a multidisciplinary experimental approach, in which a specific muscle is studied using a wide range of physiological, biochemical, structural and chemical techniques.

\section{REFERENCES}

1. Hughes VA, Frontera WR, Roubenoff R, Evans WJ, Singh MAF. Longitudinal changes in body composition in older men and women: role of body weight change and physical activity. Am J Clin Nutr. 2002;76:473-81. 
2. Baumgartner RN, Koehler KM, Gallagher D, Romero L, Heymsfield SB, Ross RR, et al. Epidemiology of sarcopenia among the elderly in New Mexico. Am J Epidemiol. 1998;147:755-63.

3. Roubenoff R. Sarcopenia and its implications for the elderly. Eur J Clin Nutr. 2000;54:S40-7.

4. Rantanen T, Guralnik JM, Foley D, Masaki K, Leveille S, Curb $\mathrm{JD}$, et al. Midlife hand grip strength as a predictor of old age disability. JAMA. 1999;281:558-60.

5. Metter EJ, Talbot LA, Schrager M, Conwit R. Skeletal muscle strength as a predictor of all-cause mortality in healthy men. J Gerontol A Biol Sci Med Sci. 2002;57:B359-65.

6. Fried LP, Guralnik JM. Disability in older adults: Evidence regarding significance, etiology, and risk. J Am Geriatr Soc. 1997;45:92-100.

7. Janssen I, Shepard DS, Katzmarzyk PT, Roubenoff R. The healthcare costs of sarcopenia in the United States. J Am Geriatr Soc. 2004;52:80-5.

8. Vandervoort AA. Aging of the human neuromuscular system. Muscle Nerve. 2002;25:17-25.

9. Szulc P, Duboeuf F, Marchand F, Delmas PD. Hormonal and lifestyle determinants of appendicular skeletal muscle mass in men: the MINOS study. Am J Clin Nutr. 2004;80:496-503.

10. Visser M, Pahor M, Taaffe DR, Goodpaster BH, Simonsick EM, Newman AB, et al. Relationship of interleukin- 6 and tumor necrosis factor-alpha with muscle mass and muscle strength in elderly men and women: The health ABC study. J Gerontol A Biol Sci Med Sci. 2002;57:M326-32.

11. Dreyer HC, Volpi E. Role of protein and amino acids in the pathophysiology and treatment of sarcopenia. J Am Coll Nutr. $2005 ; 24: 140$ S-5.

12. Thompson L, Lowe D, Ferrington D, Thomas D. Electron paramagnetic resonance: a high-resolution tool for muscle physiology. Exerc Sport Sci Rev. 2001;29:3-6.

13. Thompson LV. Effects of age and training on skeletal-muscle physiology and performance. Phys Ther. 1994;74:71-81.

14. Thompson LV. Contractile properties and protein isoforms of single skeletal muscle fibers from 12-and 30-month-old Fischer 344 Brown Norway F1 hybrid rats. Aging Clin Exp Res. 1999;11:109-18.

15. Kadhiresan VA, Hassett CA, Faulkner JA. Properties of single motor units in medial gastrocnemius muscles of adult and old rats. J Physiol. 1996;493:543-52.

16. Brooks SV, Faulkner JA. Skeletal-muscle weakness in old-age - underlying mechanisms. Med Sci Sports Exerc. 1994;26: 432-9

17. Payne AM, Delbono O. Neurogenesis of excitation-contraction uncoupling in aging skeletal muscle. Exerc Sport Sci Rev. 2004;32:36-40.

18. Li X, Larsson L. Maximum shortening velocity and myosin isoforms in single muscle fibers from young and old rats. Am J Physiol. 1996;270:C352-60.

19. Degens H, Yu F, Li X, Larsson L. Effects of age and gender on shortening velocity and myosin isoforms in single rat muscle fibres. Acta Physiol Scand. 1998;163:33-40.
20. Lowe DA, Surek JT, Thomas DD, Thompson LV. Electron paramagnetic resonance reveals age-related myosin structural changes in rat skeletal muscle fibers. Am J Physiol Cell Physiol. 2001;280:C540-7.

21. Prochniewicz E, Thomas DD, Thompson LV. Age-related decline in actomyosin function. J Gerontol A Biol Sci Med Sci. 2005;60:425-31.

22. Zhong S, Lowe DA, Thompson LV. Effects of hindlimb unweighting and aging on rat semimembranosus muscle and myosin. J Appl Physiol. 2006;101:873-80.

23. Bobkova EA, Bobkov AA, Levitsky DI, Reisler E. Effects of SH1 and SH2 modifications on myosin similarities and differences. Biophys J. 1999;76:1001-7.

24. Krivickas LS, Suh DW, Wilkins J, Hughes VA, Roubenoff R, Frontera WR. Age- and gender-related differences in maximum shortening velocity of skeletal muscle fibers. Am J Phys Med Rehabil. 2001;80:447-55.

25. D'Antona G, Pellegrino MA, Adami R, Rossi R, Carlizzi CN, Canepari $\mathrm{M}$, et al. The effect of ageing and immobilization on structure and function of human skeletal muscle fibres. J Physiol. 2003;552:499-511.

26. Larsson L, Li XP, Yu FS, Degens H. Age-related changes in contractile properties and expression of myosin isoforms in single skeletal muscle cells. Muscle Nerve. 1997; falta volume:S74-8.

27. Lowe DA, Warren GL, Snow LM, Thompson LV, Thomas DD. Muscle activity and aging affect myosin structural distribution and force generation in rat fibers. J Appl Physiol. 2004;96:498506

28. Lowe DA, Thomas DD, Thompson LV. Force generation, but not myosin ATPase activity, declines with age in rat muscle fibers. Am J Physiol Cell Physiol. 2002;283:C187-92.

29. Lowe DA, Husom AD, Ferrington DA, Thompson LV. Myofibrillar myosin ATPase activity in hindlimb muscles from young and aged rats. Mech Ageing Dev. 2004;125:619-27.

30. Harman D. Aging: a theory based on free radical and radiation chemistry. J Gerontol. 1956;11:298-300.

31. Sohal RS, Weindruch R. Oxidative stress, caloric restriction, and aging. Science. 1996;273:59-63.

32. Viner RI, Ferrington DA, Williams TD, Bigelow DJ, Schoneich C. Protein modification during biological aging: selective tyrosine nitration of the SERCA2a isoform of the sarcoplasmic reticulum $\mathrm{Ca} 2+-\mathrm{ATPase}$ in skeletal muscle. Biochem $\mathrm{J}$. 1999;340:657-69.

33. Viner RI, Williams TD, Schoneich C. Peroxynitrite modification of protein thiols: Oxidation, nitrosylation, and S-glutathiolation of functionally important cysteine residue(s) in the sarcoplasmic reticulum Ca-ATPase. Biochemistry. 1999;38:12408-15.

34. Sharov VS, Dremina ES, Galeva NA, Williams TD, Schoneich C. Quantitative mapping of oxidation-sensitive cysteine residues in SERCA in vivo and in vitro by HPLC-electrospray-tandem MS: selective protein oxidation during biological aging. Biochem J. 2006;394:605-15

35. Andrade FH, Reid MB, Allen DG, Westerblad H. Effect of hydrogen peroxide and dithiothreitol on contractile function of single skeletal muscle fibres from the mouse. J Physiol. 1998;509:565-75. 
36. Andrade FH, Reid MB, Allen DG, Westerblad H. Effect of nitric oxide on single skeletal muscle fibres from the mouse. J Physiol. 1998;509:577-86.

37. Callahan LA, She ZW, Nosek TM. Superoxide, hydroxyl radical, and hydrogen peroxide effects on single-diaphragm fiber contractile apparatus. J Appl Physiol. 2001;90:45-54.

38. Thompson LV, Durand D, Fugere NA, Ferrington DA. Myosin and actin expression and oxidation in aging muscle. J Appl Physiol. 2006;101:1581-7.

39. Syrovy I, Hodny Z. Nonenzymatic glycosylation of myosin effects of diabetes and aging. Gen Physiol Biophys. 1992;11:301-7.
40. Ramamurthy B, Hook P, Jones AD, Larsson L. Changes in myosin structure and function in response to glycation. FASEB J. 2001;15:2415-22.

41. Snow LM, Lynner CB, Nielsen EM, Neu HS, Thompson LV. Advanced glycation end product in diabetic rat skeletal muscle in vivo. Pathobiology. 2006;73:244-51.

42. Taaffe DR. Sarcopenia - exercise as a treatment strategy. Aust Fam Physician. 2006;35:130-4. 